VOL. 38 (1988) [293-298]

\title{
NEW CHARACTERISATIONS OF PSEUDOCOMPACT SPACES
}

\author{
WANG YAN-MIN
}

\begin{abstract}
In this paper, we give a new characterisation of pseudo-compact spaces, namely a space $X$ is pseudocompact if and only if each $\sigma$-point finite open cover of $X$ has a finite subfamily whose union is dense. As a corollary, we show that every pseudoconpact $\sigma$-metacompact (or screenable) space is compact, which sharpeus some known results.
\end{abstract}

\section{INTRODUCTION}

Pseudocompact spaces were defined by Hewitt in 1948. A series of equivalent conditions and interesting properties were given by Glicksberg, Smirnov, Tamano and many other authors (see $[1,6])$. Since 1951, work has been done in an attempt to determine which finite chain condition properties (such as pseudocompact, countably compact) are compatible with which covering properties (such as paracompact, metacompact, para-Lindelö) without implying compact. Aquaro proved in the mid sixties that countably compact is not compatible with meta-Lindelö; interest then turned to the question: With which covering properties is pseudocompact compatible? In 1980, Scott, Forster and Watson showed that pseudo-compact is not compatible with metacompact. In 1982, Burke and Davis [2] showed that pseudocompact is not compatible with para-Lindelöf. In 1985, Watson [8] showed that pseudocompact is compatible with meta-Lindelöf. The example in [4] showed that pseudocompact is compatible with subparacompact. But it seems to be unknown whether or not pseudocompact is compatible with $\sigma$-meta-compact (or screenable).

In this paper, we shall give an additional characterisation of pseudocompact spaces and show that pseudocompact is not compatible with $\sigma$-meta-compact.

A completely regular space is called pseudocompact if every continuous real-valued function on it is bounded (equivalently, every locally finite collection of open subsets (non-empty) is finite). A space is called Baire if no open set is the union of countably many nowhere dense subsets. As is well known, every pseudocompact space is Baire (see also [3, p. 271]. A $\pi$-base for a space $X$ is a family $\ddot{B}$ of nonempty open subsets of $X$ such that if $G$ is a nonempty open subset of $X$, then some element of $\mathcal{B}$ is contained in $G$. It is clear that the union of a $\pi$-base is dense. A space is called $\sigma$-metacompact (metacompact) if every open cover $\mathcal{U}$ has an open refinement $\mathcal{V}=\bigcup_{n=1} \mathcal{V}_{n}$ such that each $\mathcal{V}_{n}$ is point-finite ( $\mathcal{V}$ is point finite).

Received 11 December 1987

Copyright Clearance Centre, Inc. Serial-fee code: 0004-9729/88 \$A2.00+0.00. 


\section{THE MAIN RESUlts}

First state a lemma in [7].

Lemma (Watson). For any point-finite open cover $\mathcal{U}$ of a Baire space $X$, there is a $\pi$-base $\mathcal{B}$ for $X$ such that when $B \in \mathcal{B}$ and $U \in \mathcal{U}$, either $B$ is contained in $U$ or $B$ is disjoint from $U$.

A slight generalisation of Watson's lemma is the following.

LEMma. For any point-finite family $\mathcal{P}$ of open subsets of a Baire space $X$ (that is, $\mathcal{P}$ need not be a cover), there is a $\pi$-base $\mathcal{B}$ for $X$ such that when $B \in \mathcal{B}$ and $U \in \mathcal{P}$ either $B$ is contained in $U$ or $B$ is disjoint from $U$.

Proof: Clearly, $\mathcal{P}$ is a point-finite open cover of the subspace $\cup \mathcal{P}$ of $X$ and $\cup \mathcal{P}$ is Baire. So by Watson's lemma, there is a $\pi$-base $\mathcal{B}^{\prime}$ for $\cup \mathcal{P}$ such that when $B \in \mathcal{B}^{\prime}$ and $U \in \mathcal{P}$ either $B$ is contained in $U$ or $B$ is disjoint from $U$. Take a base $B^{\prime \prime}$ for the subspace $X \backslash \overline{\cup \mathcal{P}}$, then for each $B \in \mathcal{B}^{\prime \prime}, B$ is an open subset of $X$ and $B \cap U=\emptyset$ for every $U \in \mathcal{P}$. It is easy to check that $\mathcal{B}=\mathcal{B}^{\prime} \cap \mathcal{B}^{\prime \prime}$ is as required.

THEOREM 1. Let $\mathcal{V}=\bigcup_{n=1} \mathcal{V}_{n}$ be a $\sigma$-point finite open cover of a pseudocompact space $X$, then $\mathcal{V}$ has a finite subfamily whose union is dense.

Proof: Without loss of generality, let $\mathcal{V}_{n} \subseteq \mathcal{V}_{n+1}$. By virtue of the Lemma, we can find a sequence of $\pi$-bases $\mathcal{B}_{n}$ for $X$ such that when $B \in \mathcal{B}_{n}$ and $U \in \mathcal{V}_{n}$ either $B$ is contained in $U$ or $B$ is disjoint from $U$ for each positive integer $n$.

If $\mathcal{V}$ has a finite subfamily whose union is dense in $X$, then the proof is complete.

Otherwise, we can define by induction a sequence $V_{n} \in \mathcal{B}_{n}$ for each $n$, such that each $V_{n}$ is contained in $X \backslash \overline{\bigcup\left\{S t\left(V_{i}, \mathcal{V}_{i}\right): 1 \leqslant i \leqslant n-1\right\}}$. where the set $\operatorname{St}\left(V_{i}, \mathcal{V}_{i}\right)=$ $\bigcup\left\{V \in \mathcal{V}_{i}: V \cap V_{i} \neq \emptyset\right\}$.

Assume $V_{k}$ has been defined for $k \leqslant n$. Since $V_{k}$ meets at most finitely many elements of $\mathcal{V}_{k}$, the set $\bigcup_{k=1}^{n}\left\{V \in \mathcal{V}_{k}: V \cap V_{k} \neq \emptyset\right\}$ is a finite subfamily of $\mathcal{V}$, so, $X \neq$ $\overline{\bigcup \bigcup_{k=1}^{n}\left\{V \in \mathcal{V}_{k}: V \cap V_{k} \neq \emptyset\right\}=\bigcup_{n=1}^{n} S t\left(V_{k}, \mathcal{V}_{k}\right)}$, that is $X \backslash \overline{\bigcup_{k=1}^{n} S t\left(V_{k}, \mathcal{V}_{k}\right)}$ is a nonempty open subset. Hence there is $V_{n+1} \in \mathcal{B}_{n+1}$ such that $\emptyset \neq V_{n+1} \subseteq X \backslash \overline{\bigcup_{k=1}^{n} S t\left(V_{k}, V_{k}\right)}$ since $\mathcal{B}_{n+1}$ is a $\pi$-base. So $V_{n+1}$ is defined. Now we show that $\left\{V_{n}\right\}_{n+1}^{\infty}$ is a locally finite family of nonempty open subsets. For each $x \in X$, there is an $n_{o}$ and a $V \in \mathcal{V}_{n_{0}}$ with $x \in V$. We have to show that $V$ meets at most finitely many elements of $\left\{V_{n}\right\}_{n=1}^{\infty}$. In fact, if $V \cap V_{k} \neq \emptyset$ (we can assume $k>n_{0}$, otherwise the proof is complete), then for every $n>k$, we have $V \cap V_{n}=\emptyset$ since $V \subseteq S t\left(V_{k}, \mathcal{V}_{k}\right) \subseteq \bigcup_{1 \leqslant i<n} S t\left(V_{i}, \mathcal{V}_{i}\right)$ (note 
$\left.\mathcal{V}_{n} \subseteq \mathcal{V}_{n+1}\right)$, but $V_{n} \subseteq X \backslash \bigcup_{i=1}^{n-1} S t\left(V_{i}, \mathcal{V}_{i}\right)$ : that is, $V$ meets at most finitely many elements of $\left\{V_{n}\right\}_{n=1}^{\infty}$. But $X$ is pseudocompact, so there is no infinite family of open sets which is locally finite. This is a contradiction. So there are finitely many elements of $\mathcal{V}$ whose union is dense in $X$.

THEOREM 2. Every pseudocompact $\sigma$-metacompact space is compact.

Proof: Let $\mathcal{O}$ be an open cover of $X$ and $\mathcal{V}=\bigcup_{n=1}^{\infty} \mathcal{V}_{n}$ be an open refinement such that each $\mathcal{V}_{n}$ is point-finite. Using regularity, we can assume that if $U \in \mathcal{V}$, then the closure of $U$ is contained in some element of $\mathcal{O}$. By Theorem 1, $\mathcal{V}$ has a finite subfamily whose union is dense; hence $\mathcal{O}$ has a finite subcover.

Recall that a space $X$ is called screenable if each open cover of $X$ has a $\sigma$-disjoint open refinement.

COROLLARY 1. Every pseudocompact screenable space is compact.

Corollary 2. (Scott, Forster, Watson). Every pseudocompact metacompact space is compact.

Example 1. There exists a Lindelöf (hence screenable, $\sigma$-metacompact) Hausdorff space which is not metacompact.

Let $\mathcal{T}$ denote the usual topology on the closed interval $[0,1]$, let $\mathcal{B}=\{V \backslash A: V \in$ $\mathcal{T}, A \subseteq Q\}$ where $Q$ is the set of all rationals in $[0,1]$. Let $X$ be the new space with the topology $\mathcal{B}$. It is clear that $X$ is a Lindelöf (even hereditarily Lindelöf), Hausclorff (since $\mathcal{B}$ is finer than $\mathcal{T}$ ) space.

It remains to show that $X$ is not metacompact.

Lemma. Let $X$ be metacompact, then each directed open cover $\mathcal{U}$ (that is, for each pair $V_{1}, V_{2} \in \mathcal{U}$, there is a $V_{3} \in \mathcal{U}$ with $V_{3} \supseteq V_{1} \cup V_{2}$ ) has a closure-preserving closed refinement.

Proof: Let $\mathcal{U}$ be a directed open cover of $X$ and $\mathcal{V}$ be its point-finite open refinement. Let $\mathcal{V}^{F}$ denote the collection of all unions of finite subfamily of $\mathcal{V}$, then $\mathcal{V}^{F}$ also is an open refinement of $\mathcal{U}$ (not necessarily point-finite). For $G \in \mathcal{V}^{F}$, let $F_{G}=\{x \in X: S t(x, \mathcal{V}) \subseteq G\}$, then the collection $\mathcal{F}=\left\{F_{G} \mid G \in \mathcal{V}^{F}\right\}$ is a closurepreserving closed refinement of $\mathcal{V}^{F}$ (hence, of $\mathcal{U}$ ). In fact:

(1) For each $G \in \mathcal{V}^{F}, F_{G}$ is a closed set. Since if $x \notin F_{G}$, then $\operatorname{St}(x, \mathcal{V}) \nsubseteq$ $G$, equivalently, there is a $V \in \mathcal{V}$ with $x \in V$ such that $V \nsubseteq G$, Hence $V \cap F_{G}=\emptyset$; that is, $F_{G}$ is a closed set.

(2) $\mathcal{F}$ is a cover. For each $x \in X$, let $\mathcal{V}_{x}=\{V \in \mathcal{V}: x \in V\}$. Then $\mathcal{V}_{x}$ is a finite subfamily so $U \mathcal{V}_{x} \in \mathcal{V}^{F}, x \in F_{\cup \mathcal{V}_{x}}$. 
(3) $\mathcal{F}$ is closure-preserving.

Let $\mathcal{W}$ be a subset of $\mathcal{V}^{F}$. If $x \notin \cup\left\{F_{G}: G \in \mathcal{W}\right\}$, let $V_{x}=\cap\{V \in \mathcal{V}: x \in V\}$ then we have $V_{x} \cap\left(\cup\left\{F_{G}: G \in \mathcal{W}\right\}\right)=\emptyset$, because if $y$ is in $V_{x} \cap\left(\cup\left\{F_{G}: G \in \mathcal{W}\right\}\right)$, then there is a $G_{0} \in \mathcal{W}$ such that $y \in F_{G_{0}}$, so $\operatorname{St}(x, \mathcal{V})=\cup \mathcal{V}_{x} \subseteq \operatorname{St}(y, \mathcal{V}) \subseteq G_{0}$; equivalently, $x \in F_{G_{0}}$. This contradiction proves the lemma.

To show that $X$ is not metacompact, we still need some observations. First, we note that every closed subset $F$ of $X$ can be written in the form $E \cup A$, where $E$ is closed in the standard topology and $A \subseteq Q$. Then, we also note that every nowhere dense subset $D$ in $X$ can be expressed as the union of a standard nowhere dense subset and a subset of $Q$. Thus we have that $X$ cannot be expressed as a union of countably many nowhere dense subsets, that is, $X$ is Baire. Now we can easily show that $X$ is not metacompact. Otherwise, let $Q=\left\{r_{1}, r_{2}, \ldots\right\}, U_{0}=[0,1] \backslash Q, U_{n}=U_{0} \cup\left\{r_{1}, \ldots r_{n}\right\}$, for each $n$, and $\mathcal{U}=\left\{U_{n}\right\}_{n=1}^{\infty}$, then $\mathcal{U}$ is an open cover of $X$. So there is a closurepreserving closed refinement $\mathcal{F}$ of $\mathcal{U}$ since $\mathcal{U}$ is directed. Without loss of generality, we can assume $\mathcal{F}=\left\{F_{n}\right\}_{n=1}^{\infty}$ such that each $F_{n}$ is closed and $F_{n} \subseteq U_{n}$. Hence we can fincl an $F_{i}^{0} \neq \emptyset$ for some $i \in N$ by the above observation. Let $F_{i}^{0}=U=V \backslash A$, where $V$ is a standard open subset and $A \subseteq Q$. Noting that $\widetilde{U}=\bar{V}=\bar{U}$, where ' $\sim$ ' and '-'denote the new closure operation and the standard one, respectively. So $F_{i}=\widetilde{F}_{i} \supseteq \widetilde{U}=\bar{V}$, that is $F_{i}$ contains infinitely many rationals. This contradicts the fact that $F_{i} \subseteq U_{i}=([0,1] \backslash Q) \cup\left\{r_{1}, r_{2}, \ldots r_{i}\right\}$, which shows that $X$ is indeed not metacompact.

Remark. In the above example, the separation assumption cannot be strengthened, since it is well-known that every Lindelof regular space is paracompact. If we assume extra set-theoretic axioms, there is a normal screenable space which is not metacompact.

Example 2. (Rudin). Assume $V=L$, there is a normal screenable non-metacompact space $([\mathbf{5}])$.

THEOREM 3. For any space $X$, the following assertions are equivalent:

(1) $X$ is pseudocompact;

(2) every locally finite family of non-empty open sets of $X$ is finite;

(3) every locally finite open cover of $X$ has a finite subcover;

(4) every locally finite functional open cover of $X$ has a finite cover;

(5) every locally finite functional open cover of $X$ has a finite sub-family whose union is dense;

(6) every countable, locally finite functional open cover of $X$ has a finite subfamily whose union is dense;

(7) every countable, locally finite functional open cover of $X$ has a finite subcover; 
(8) every $\sigma$-point-finite open cover of $X$ has a finite subfamily whose union is dense.

Proof: $(1) \Leftrightarrow(2) \Leftrightarrow(3)$ are well known, $(3) \Rightarrow(4) \Rightarrow(5) \Rightarrow(6)$ and $(8) \Rightarrow$ (6) are clear.

$(6) \Rightarrow(7)$ : We need note only that every locally finite functionally open cover of a topological space is shrinkable (also see [3, p. 481, 7.1.B)].

$(\tau) \Rightarrow(1)$ : Clearly, the family $\left\{f^{-1}(i-1, i+1): i=0, \pm 1, \pm 2, \ldots\right\}$ is a countable, locally finite open cover of $X$; the existence of a finite subcover implies that $f$ is bounded.

(1) $\Rightarrow$ (8): See Theorem 1 .

Remark. In the proof of Theorem 1, the assumption of complete regularity is not necessary. Recall that a space is lightly compact if each locally finite family of nonempty open sets is finite ( see [1]). It is clear that $X$ is lightly compact if and only if for each decreasing sequence $W_{1} \supseteq W_{2} \ldots$ of non-empty open subsets of $X$ the intersection $\bigcap_{n=1}^{\infty} \bar{W}_{i}$ is non-empty. We also have that

Lemma. A regular lightly compact space is Baire.

The proof is clear.

Theorem 4. A lightly compact $\sigma$-metacompact regular space is compact.

Theorem 5. For a Baire space $X, X$ is lightly compact, and $\sigma$-metacompact if and only if $X$ is compact.

A lightly compact, Baire space also has a series of equivalent conditions similar to those of Theorem 3 . We leave these to the reader as exercises.

\section{REFERENCES}

[1] R.W. Bagley, E.H. Connell and J.D. McKnight, Jr, 'On properties characterising pseudocompact spaces', Proc. Amer. Math. Soc. 9 (1958), 500-506.

[2] D.A. Burke and S.W. Davis, 'Pseudocompact paralindelof spaces are compact', Abstracts Amer. Math. Soc. 3 (1982), p. 213.

[3] R. Englking, General Topology (Warszawa, 1977).

[4] L. Gillman and M. Jerison, Rings of continuous functions: Graduate Texts in Math. 43 (Springer, Berlin, 1960).

[5] M.E. Rudin, 'A normal screenable non-paracompact space', Topology Appl. 15 (1983), p. 313.

[6] R.M. Stephenson, 'Pseudocompact spaces', Trans. Amer. Math. Soc. 134 (1968), 436 448.

[7] W.S. Watson, 'Pseudocompact metacompact spaces are compact', Proc. Amer. Math. Soc. 81 (1981), P. 151.

[8] W.S. Watson, 'Pseudocompact meta-Lindelöf space which is not compact', Topology Appl. 20 (1985), 237-243. 
Department of Basic Sciences

Shanghai Institute of Mechanical Engineering

Shangliai

Peoples Republic of China 